\title{
Severe panuveitis in neuro-Behçet's disease in Malaysia: a case series
}

This article was published in the following Dove Press journal:

International Medical Case Reports Journal

7 February 2017

Number of times this article has been viewed

\author{
Khairuddin Othman' \\ Ahmad Tajudin \\ Liza-Sharmini' \\ Mohtar Ibrahim' \\ John Tharakan ${ }^{2}$ \\ Ryoji Yanai ${ }^{3}$ \\ Embong Zunaina' \\ 'Department of Ophthalmology, \\ ${ }^{2}$ Department of Neurosciences, \\ School of Medical Sciences, Universiti \\ Sains Malaysia, Kubang Kerian, \\ Kelantan, Malaysia; ${ }^{3}$ Department of \\ Ophthalmology, Yamaguchi University \\ Graduate School of Medicine, Ube, \\ Yamaguchi, Japan
}

\begin{abstract}
Behçet's disease (BD) is a multisystemic disease that is very rare in Malaysia. About $5 \%$ of patients develop central nervous system involvement, termed neuro-Behçet's. NeuroBehçet's is one of the most serious causes of long-term morbidity and mortality. We report two cases of neuro-Behçet's associated with uveitis (ocular BD) highlighting the clinical presentation, diagnostic measurement, and therapeutic management of these cases.
\end{abstract}

Keywords: Behçet's disease, Behçet's syndrome, parenchymal neuro-Behçet's, uveitis

\section{Introduction}

Behçet's disease (BD) is a multisystemic, chronic relapsing inflammatory disease classified as one of the vasculitides. ${ }^{1,2}$ The pathogenesis is unclear but histopathologically suggestive of occlusive vasculitis. ${ }^{3-5} \mathrm{BD}$ was first described by a dermatologist in patients with the triad of recurrent severe uveitis and painful oral and genitalia ulcers. ${ }^{3}$ BD can be further divided into three subtypes: neuro-Behçet's, entero-Behçet's, and vasculo-Behçet's. ${ }^{4}$ Neuro-Behçet's can be classified further into acute and chronic progressive type. Acute neuro-Behçet's is characterized by remission and exacerbation with good response to corticosteroids therapy. ${ }^{5,6}$ Chronic progressive neuro-Behçet's is intractable and resistant to corticosteroid, cyclophosphamide and azathioprine (AZT) ${ }^{5-7}$ On the basis of the involvement of the brain, it is divided into parenchymal; focal or multifocal brain parenchyma involvement and nonparenchymal; major cerebral vessels involvement. ${ }^{5,8-10}$

$\mathrm{BD}$ is reported worldwide but is more prevalent in the Eastern Mediterranean countries, Middle-East, and Japan. ${ }^{11-13}$ To the best of our knowledge, only one case of BD with vascular involvement has been reported in Malaysia. ${ }^{4}$ No case of neuroBehçet's has ever been reported in Malaysia. We reported two cases of debilitating neuro-Behçet's with severe panuveitis in Malaysian patients.

\section{Case report Case I}

A 47-year-old man presented to the ophthalmology clinic in 2001 with bilateral red eyes for 4 days duration. He also complained of eye pain, photophobia, floaters, and blurring of vision. He had $\sim 5-6$ similar attacks of red eye for the past 4 years. Each attack of red eye gradually resolved over 2-4 weeks duration. Systemically, he had recurrent episodes of painful oral and genital ulcers, which resolved spontaneously after several days.
Department of Ophthalmology, School of Medical Sciences, Universiti Sains Malaysia, Kubang Kerian 16150, Kelantan, Malaysia

Tel +6097676362

Fax +6097653370

Email zunaina@usm.my 
On ocular examination, the vision was $1 / 60$ in the right eye and hand movement in the left eye. Both eyes showed features of nongranulomatous panuveitis, perilimbal injection, keratic precipitates, and moderate anterior chamber reaction with $1 \mathrm{~mm}$ hypopyon level in the left eye. There was presence of moderate vitritis with multiple areas of retinal infiltrates bilaterally and multiple retinal hemorrhages in the left eye. Intraocular pressure (IOP) was not elevated. An infective and connective tissue screening was normal except for an elevated erythrocyte sedimentation rate (ESR; $68 \mathrm{~mm} /$ hour).

A diagnosis of $\mathrm{BD}$ was made based on the clinical triad of uveitis, and oral and genital ulcers. He was treated with oral prednisolone $80 \mathrm{mg}$ daily $(1.5 \mathrm{mg} / \mathrm{kg} /$ day $)$ and topical prednisolone acetate $1 \% 4$-hourly for both eyes. The oral prednisolone was tapered down $5 \mathrm{mg}$ per week. At 6 weeks on tapering dose of oral prednisolone, his vision improved to $6 / 12$ in the right eye and 1/60 in the left eye with resolving of the ocular inflammation clinically. However, he defaulted follow-up.

In 2013, he presented to the outpatient clinic with left shoulder pain for 6 months duration. There was also left upper limb weakness and limited range of movement of his shoulder. Prior to that, the patient had recurrent episodes of right upper limb weakness and pain, which recovered fully without treatment. The left shoulder pain resolved gradually with analgesics and physiotherapy. However, during the course of physiotherapy, he defaulted again.

He came back again 2 years later to the neurology clinic with unsteady gait and difficulty in walking for the past 1 year. It was also associated with hypophonia and dysphagia. However, there was no upper limbs pain or weakness. Neurological examination revealed pyramidal weakness of both upper and lower limbs with involvement of IX, X, and XII cranial nerves. There was also presence of oral ulcers (Figure 1). However, there was no genital ulcer and the pathergy test was negative. He was then referred to ophthalmology team for further eye assessment. There was no specific ocular symptom except for painless progressive blurring of vision of both eyes. His vision deteriorated to perception to light bilaterally, with old keratic precipitates and an immature cataract noted. Fundus examination showed bilateral optic atrophy with attenuated retinal vessels and pigmentary changes of the retina.

$\operatorname{ESR}(73 \mathrm{~mm} /$ hour $)$ and C-reactive protein $(25 \mathrm{mg} / \mathrm{L})$ were elevated. Magnetic resonance imaging (MRI) of the brain showed small round multiple hyperintense lesions at both frontal lobes on T2-weighted and fluid-attenuated inversion

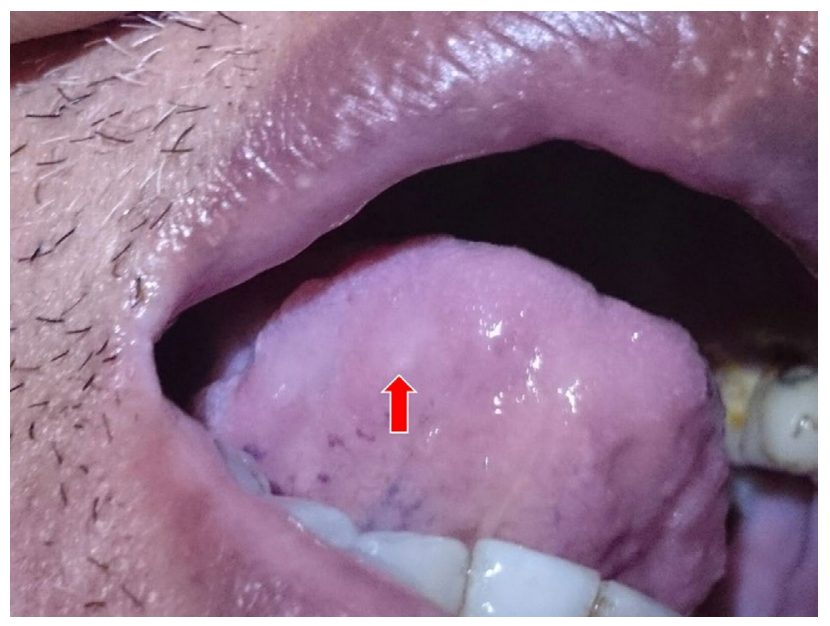

Figure I Oral ulcer at the posterior aspect of the tongue (red arrow).

recovery images (Figure 2). The lesions were absent on T1-weighted image, suggestive of acute or subacute lesion of the brain. However, he refused lumbar puncture procedure for cerebrospinal fluid (CSF) analysis.

He was diagnosed to have neuro-Behçet's clinically. $\mathrm{He}$ was treated with intravenous methylprednisolone $1 \mathrm{~g}$ per day for 5 days then continued with oral prednisolone $40 \mathrm{mg}$ daily. After 3 months, oral AZT $25 \mathrm{mg}$ daily was added and the oral prednisolone was tapered down slowly to a maintenance dose of $20 \mathrm{mg}$ daily. There was no relapse of disease and no progression of his neurological deficit after almost 1 year of treatment. He is ambulating well with walking frame. The summary of clinical presentation and treatment is shown in Table 1.

\section{Case 2}

A 25-year-old active young man presented to emergency medicine and trauma care in June 2014 for progressive bilateral lower limbs weakness and spasticity for the past 6 months. His lower limbs weakness and spasticity were initially episodic with full recovery without treatment. Later it became more frequent and affected his daily activity. He also complained of urinary hesitancy and recurrent multiple painful genitalia and oral ulcers. He was treated with oral carbamazepine $200 \mathrm{mg}$ daily for lower limbs spasticity. Subsequently, he developed cerebellar signs, dysarthria, nystagmus, and intentional tremor. MRI of brain and spine was performed to rule out compressive myelopathy and the result was normal. Blood investigations for sexual communicable diseases and autoimmune diseases were negative. Based on clinical finding and inconclusive investigations, he was diagnosed as primary spinocerebellar degeneration with a differential diagnosis of vasculitic Behçet's. Despite 
A

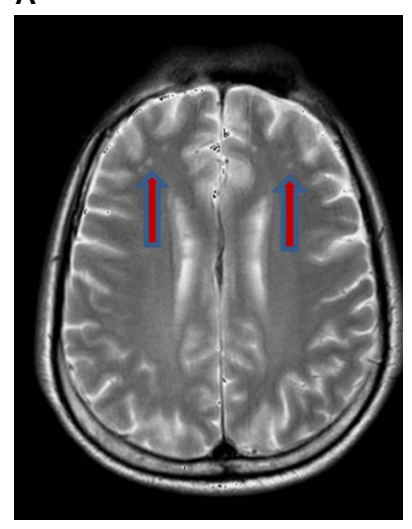

B

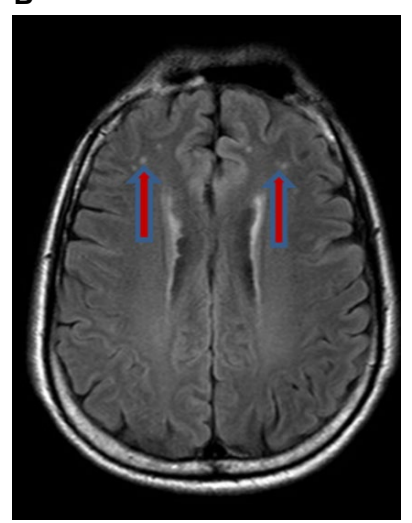

C

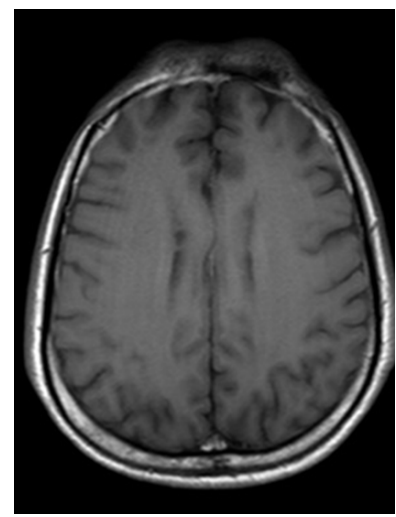

Figure 2 Magnetic resonance imaging of the brain showed small round multiple hyperintense lesions at bifrontal lobes on T2-weighted (A) and fluid-attenuated inversion recovery images (B) (red arrows). The lesions were not seen on TI-weighted image (C)

Table I Summary of clinical presentation and treatment for both cases

\begin{tabular}{|c|c|c|}
\hline Cases & Clinical presentation & Treatment \\
\hline Case I & 47-year-old man & \\
\hline \multirow[t]{2}{*}{2001} & Behçet's disease with bilateral panuveitis & Oral prednisolone $80 \mathrm{mg}$ daily $(1.5 \mathrm{mg} / \mathrm{kg} /$ day $)$ with tapering dose $5 \mathrm{mg} /$ week \\
\hline & & Topical prednisolone acetate $1 \% 4$-hourly for both eyes \\
\hline $2002-2012$ & Defaulted follow-up & No treatment \\
\hline \multirow[t]{2}{*}{2013} & Upper limb weakness & Analgesic \\
\hline & & Physiotherapy \\
\hline 2014 & Defaulted follow-up & No treatment \\
\hline \multirow[t]{5}{*}{$2015-2016$} & Neuro-Behçet's with bilateral optic atrophy & Intravenous methylprednisolone I g/day for 5 days followed by oral \\
\hline & & prednisolone $40 \mathrm{mg}$ daily for 3 months \\
\hline & & Then oral prednisolone was tapered down slowly to a maintenance dose of \\
\hline & & 20 mg daily \\
\hline & & Oral azathioprine $25 \mathrm{mg}$ daily started during tapering dose of oral prednisolone \\
\hline Case 2 & 25-year-old man & \\
\hline 2014 & Primary spinocerebellar degeneration & Oral carbamazepine $200 \mathrm{mg}$ daily for lower limbs spasticity \\
\hline \multirow[t]{7}{*}{$2015-2016$} & Neuro-Behçet's with bilateral panuveitis complicated & Intravenous methylprednisolone I g/day for 5 days followed by oral \\
\hline & with neovascular glaucoma in the right eye & prednisolone $50 \mathrm{mg}$ daily \\
\hline & & Then oral prednisolone was tapered down slowly \\
\hline & & Topical antiglaucoma for high intraocular pressure \\
\hline & Blebitis post-trabeculectomy and subsequently & Intravenous, intravitreal, and topical antibiotic \\
\hline & developed endophthalmitis of the right eye & \\
\hline & Recurrent attacks of posterior uveitis of the left eye & Higher dose of oral prednisolone and addition of oral cyclosporine \\
\hline
\end{tabular}

A

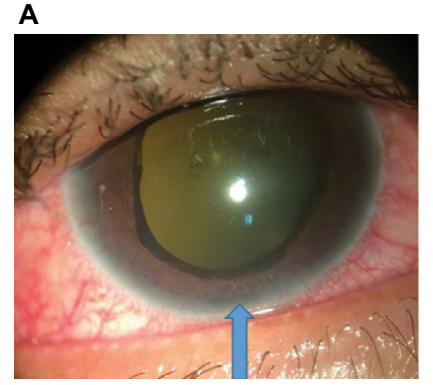

B

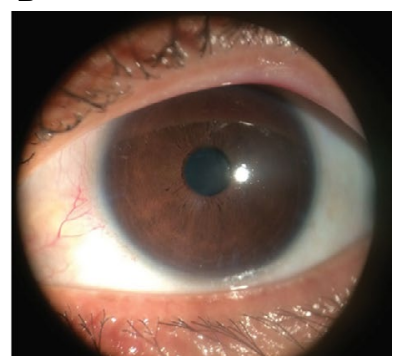

Figure 3 Right eye showed rubeosis iridis with ectropion uvea and hypopyon (blue arrow) (A) and mild anterior segment inflammation in the left eye (B).

being on treatment, his limb weakness progressed. He was paraplegic and wheel chair dependent.

One year after his initial presentation, he developed painful right eye with reduction of vision for 3 weeks. He was then referred to ophthalmology team for further eye assessment. Retrospectively, he had history of right eye painless progressive blurring of vision for the past 1 year. His vision was perception to light on the right eye while left eye vision was $6 / 12$. Ocular examination showed the presence of bilateral uveitis. Anterior segment of the right eye revealed severe anterior chamber inflammation with the presence of hypopyon, 360 degree rubeosis iridis with ectropion uvea (Figure $3 \mathrm{~A}$ ), and dense diffuse vitritis that impaired the visualization of the fundus. There was mild anterior segment inflammation in the left eye (Figure 3B) with the presence of mild vitritis and vitreous opacities. There was no vasculitis, retinitis, or retinal hemorrhage and exudate seen in the left eye. Right eye was complicated with neovascular glaucoma with IOP of $44 \mathrm{mmHg}$. IOP of left eye was $14 \mathrm{mmHg}$. 
Further assessment was performed to support the diagnosis of BD; pathergy test positive (Figure 4). Screening for infective and connective tissue diseases was negative. Another MRI of the brain revealed atrophy of the cerebellar and frontal lobe (Figure 5). He refused lumbar puncture. He was diagnosed as neuro-Behçet's based on progressive neurological deficits of the lower limbs, recurrent multiple painful genitalia and oral ulcers, bilateral uveitis, and positive pathergy test. He was then treated with intravenous methylprednisolone $1 \mathrm{~g}$ daily for 5 days followed by oral prednisolone $50 \mathrm{mg}$ daily. His IOP was not controlled with antiglaucoma drugs, and right eye augmented trabeculectomy was performed (Figure 6A and B). However, the bleb site was

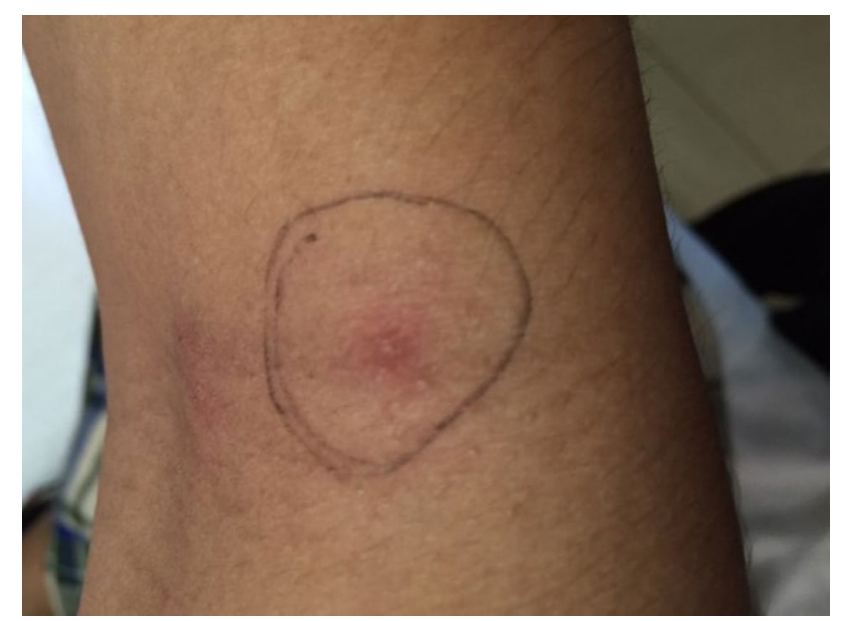

Figure 4 Positive pathergy test showed pustular lesion following 24-48 hours of 23-gauge sterile needle injected subcutaneously.

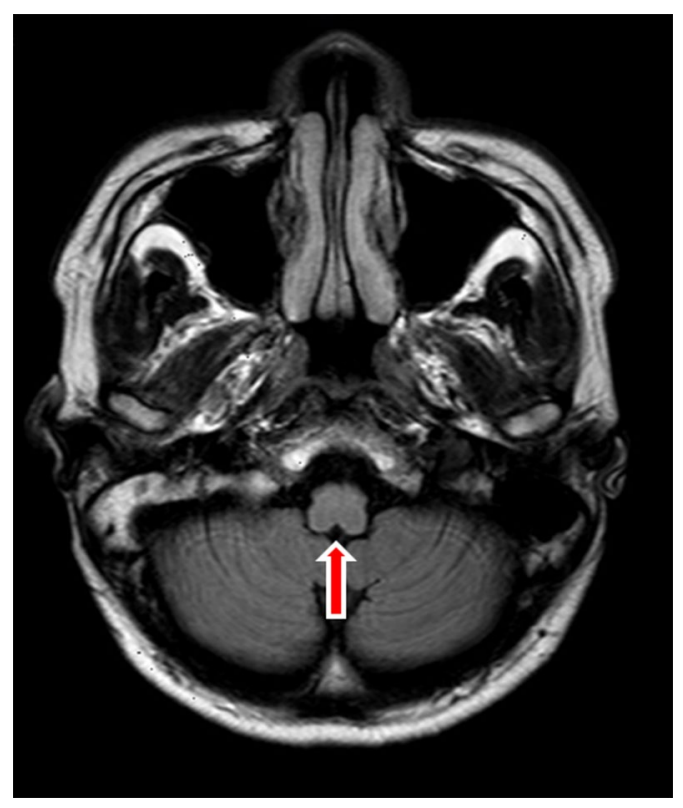

Figure 5 Magnetic resonance imaging of the brain showed atrophy of the brainstem (red arrow).
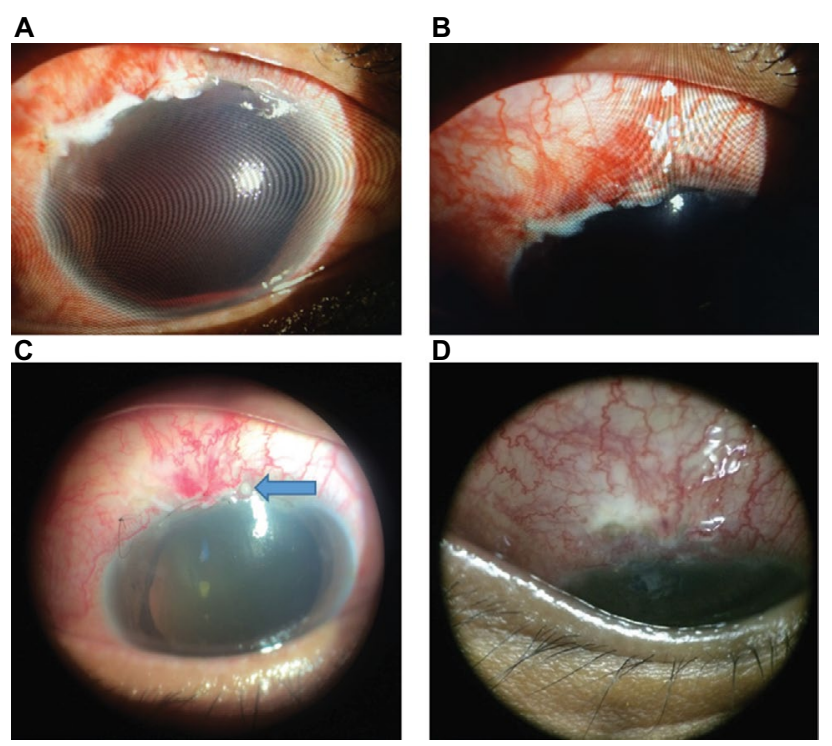

Figure 6 Right eye post-trabeculectomy (A) with close-up view (B), complicated with suture abscess (blue arrow) (C) and followed with blebitis (D).

complicated with suture abscess (Figure 6C) and followed with blebitis (Figure 6D), then subsequently developed endophthalmitis. In view of that, the oral prednisolone was tapered down slowly. Despite being on intravenous, intravitreal, and topical antibiotic, the right eye progressed to painless blind eye. There was no progression of neurological deficit throughout follow-up. However, he continued to develop recurrent attacks of posterior uveitis of the left eye. Ocular inflammation was well controlled with higher doses of oral prednisolone and addition of oral cyclosporine. The summary of clinical presentation and treatment is shown in Table 1.

\section{Discussion}

Neuro-Behçet's is rare, yet the most debilitating complication of $\mathrm{BD}$. The incidence was reported as $4 \%-59 \%$ among BD. ${ }^{14-18}$ Unlike BD, neuro-Behçet's is more common in men than in women with a male-to-female ratio of $3.4: 1$ to $4: 1$. $^{8,17}$ Our reported case series involved young to middle-aged Asian men. Commonly neurological manifestations develop after several years of development of BD, ranging from 3 to 6 years. ${ }^{5,8,9}$ The onset of neuro-Behçet's was rather delayed in case 1, 12 years after the presentation of recurrent uveitis and ulcers. Joseph and Scolding reported a mean duration of 12 years from the onset of BD in 22 cases of neuro-Behçet's. ${ }^{19}$ There were differences in the clinical presentation of both cases, but both fulfilled the criteria of neuro-Behçet's based on International Consensus Recommendation (ICR) criteria for neuro-Behçet's diagnosis. ${ }^{20}$

However, in $6 \%$ of cases of neuro-Behçet's, neurological manifestation precedes the systemic symptoms of $\mathrm{BD} .{ }^{8}$ This 
may perhaps lead to difficulty in diagnosis, as illustrated in case 2 . The presence of urinary hesitancy and genital ulcers may lead to other possible diagnoses, especially in a young healthy man. Spinal and cerebellar sign and symptoms may easily mimic spinocerebellar degeneration or multiple system atrophy. ${ }^{21}$ Diagnosis of neuro-Behçet's is difficult in nonestablished BD.

$\mathrm{BD}$ is rare in Malaysia, and no case of neuro-Behçet's has ever been reported in Malaysia. We reported two cases of neuro-Behçet's who presented with ocular involvement at different courses of the disease. Based on ICR criteria, case 1 is a case of definite neuro-Behçet's and case 2 is probable neuro-Behçet's at the beginning of the course of the disease. Ocular involvement was at the initial course of the disease in case 1 , while in case 2 , it was at the later stage of the disease. However, complications of severe ocular inflammation resulted in visual impairment and blindness in both cases. The most common ocular manifestation in $\mathrm{BD}$ is retinal vessel inflammatory changes, periphlebitis and periarteritis, serofibrinous anterior uveitis, and panuveitis. ${ }^{22-27}$

In both cases, although both eyes were affected, ocular manifestations were more severe in one eye. Nongranulomatous panuveitis was seen in both cases, with retinal inflammation. Case 2 presented with complications of severe uveitis, neovascular glaucoma, and cataract during the initial presentation. The Systemic Immunosuppressive Therapy for Eye Disease Cohort study reported that $10 \%$ of BD with posterior ocular involvement developed cataract compared with $6 \%$ of the cases with anterior uveitis, while secondary glaucoma is observed in $19 \%$ of $\mathrm{BD}$ with anterior uveitis, and only $13 \%$ of $\mathrm{BD}$ with posterior or panuveitis. ${ }^{24}$ Elgin et al reported that $\sim 11 \%$ of $\mathrm{BD}$ patients developed secondary glaucoma. Half of the secondary glaucoma cases were steroid- or inflammation-induced open-angle glaucoma, while only $10 \%$ developed neovascular glaucoma. ${ }^{28}$

Addressing elevation of IOP in cases of BD with neovascular glaucoma is always frustrating. Neovascular glaucoma is a refractory glaucoma that usually requires surgical intervention, glaucoma drainage device implantation, or augmented trabeculectomy. On the other hand, the inflammation in $\mathrm{BD}$ requires treatment with high dose of systemic steroids or other immunosuppressive treatment. ${ }^{27,29,30} \mathrm{Immu}-$ nosuppressive treatment increases the risk of infection. In case 2, patient developed endophthalmitis after augmented trabeculectomy and lost his vision.

To date, there is no reported study on the relationship of severe ocular inflammation and neuro-Behçet's. In addition, there was no single report on the specific test able to predict the manifestation of neuro-Behçet's in BD patients. Based on their clinical presentation, both cases presented with parenchymal neuro-Behçet's. Parenchymal neuro-Behçet's is characterized by focal or multifocal involvement of brain parenchyma, leading to hemiparesis, corticospinal tract or pyramidal signs, brainstem, and cerebellar signs. ${ }^{8,9,20,31-33}$

MRI findings in neuro-Behçet's include small focal or multifocal lesions of high signal intensity on T2-weighted images (Figure 2) and iso- or hypointense lesions on T1-weighted images. ${ }^{2,20}$ The lesion occurs most frequently within the brainstem, followed by thalamus and basal ganglia. ${ }^{2}$ The presence of brainstem-thalamic-basal ganglia lesions can strongly support the diagnosis of acute/subacute parenchymal neuro-Behçet's and raise the possibility of BD when the systemic features are scarce. ${ }^{34}$ This lesion represents inflammatory cellular infiltration, demyelination, and edema, which may diminish post-treatment with corticosteroid and/ or immunosuppressive agents. ${ }^{2}$ In chronic disease as in case 2, atrophic lesions can be found in brainstem (Figure 4).

Pathergy test may perhaps assist in making the definitive diagnosis. Although positive pathergy test is one of the criteria for diagnosis of $\mathrm{BD}$, it is not pathognomonic of BD. ${ }^{20}$ Pathergy test was negative in case 1 but positive in case 2 . Other test includes CSF examination and major histocompatibility complex human leukocyte antigen (HLA) B-5 allele and HLA B-51.

Till date no controlled or comparative trials have evaluated treatment of neuro-Behçet's. Both of our patients received intravenous methylprednisolone $1 \mathrm{~g}$ /day for 5 days followed by maintenance oral corticosteroids. ICR recommended early consideration of a disease-modifying therapy such as AZT when one or more poor prognostic features are encountered. Poor prognostic factors include brainstem or spinal cord presentation, frequent relapses, early disease progression, and high CSF pleocytosis. ${ }^{8,17,31}$ AZT was added to systemic steroids because of frequent relapses in case 1. Both cases resulted in visual impairment and blindness with neurological deficit. BD and neuro-Behçet's patients should understand their course of the disease, especially the symptoms of ocular and systemic relapse, to avoid delay in seeking medical attention.

\section{Conclusion}

It is important to consider neuro-Behçet's in neurological patients with unexplained findings. Detection and thorough investigation for any nongranulomatous uveitis may help in early diagnosis of BD and neuro-Behçet's. Creating awareness and better understanding of BD and neuro-Behçet's may prevent ocular and systemic complications. 


\section{Acknowledgment}

The authors would like to thank both of the patients who provided their written informed consent to participate in this case series and for the publication of their photos.

\section{Disclosure}

The authors report no conflicts of interest in this work.

\section{References}

1. Deepak B, Jyotirmay B. Successful treatment of Behcet's eye disease with infliximab therapy. J Ophthalmic Clin Res. 2015;2(1):1-3.

2. Banna M, El-Ramahl K. Neurologic involvement in Behçet disease: imaging findings in 16 patients. AJNR Am J Neuroradiol. 1991;12(4): 791-796.

3. Huston KA. Behcet's disease: a contemporary synopsis. Paper presented at: Mayo Clinic Proceedings; 1989; MN.

4. Normayah K, Mazri YM, Suib I, Zainal AA. Behcet's disease with vascular complications. Med J Malaysia. 2004;59(4):547-549.

5. Al-Araji A, Kidd DP. Neuro-Behçet's disease: epidemiology, clinical characteristics, and management. Lancet Neurol. 2009;8(2):192-204.

6. Hirohata S. Potential new therapeutic options for involvement of central nervous system in Behçet's disease (neuro-Behçet's syndrome). Curr Rheumatol Rev. 2007;3(4):297-303.

7. Matsui T, Ishida T, Tono T, Yoshida T, Sato S, Hirohata S. An attack of acute neuro-Behçet's disease during the course of chronic progressive neuro-Behçet's disease: report of two cases. Mod Rheumatol. 2010; 20(6):621-626.

8. Akman-Demir G, Serdaroglu P, Tasçi B. Clinical patterns of neurological involvement in Behçet's disease: evaluation of 200 patients. Brain. 1999;122(11):2171-2182.

9. Siva A, Altintas A, Saip S. Behçet's syndrome and the nervous system. Curr Opin Neurol. 2004;17(3):347-357.

10. Inaba G. Proceedings of the International Conference on Behçet's Disease : Behçet's Disease - Pathogenetic Mechanism and Clinical Future: October 23-24, 1981. Tokyo: University of Tokyo Press; 1982.

11. Verity DH, Marr JE, Ohno S, Wallace GR, Stanford MR. Behçet's disease, the Silk Road and HLA-B51: historical and geographical perspectives. Tissue Antigens. 1999;54(3):213-220.

12. Pande I, Uppal SS, Kailash S, Kumar A, Malaviya AN. Behcet's disease in India: a clinical, immunological, immunogenetic and outcome study. Br J Rheumatol. 1995;34(9):825-830.

13. Gürler A, Boyvat A, Türsen U. Clinical manifestations of Behçet's disease: an analysis of 2147 patients. Yonsei Med J. 1997;38(6): 423-427.

14. Fadli ME, Youssef MM. 'Neuro-Behçet's syndrome in the United Arab Republic. Eur Neurol. 1973;9(2):76-89.
15. Shimizu T, Ehrlich GE, Inaba G, Hayashi K. Behçet disease (Behçet syndrome). Semin Arthritis Rheum. 1979;8(4):223-260.

16. Serdaroğlu P, Yazici H, Özdemir C, Yurdakul S, Bahar S, Aktin E. Neurologic involvement in Behçet's syndrome: a prospective study. Arch Neurol. 1989;46(3):265-269.

17. Siva A, Kantarci OH, Saip S, et al. Behçet's disease: diagnostic and prognostic aspects of neurological involvement. J Neurol. 2001;248(2): 95-103.

18. Ben Taarit, Turki S, Ben Maiz H. Neurological manifestations in Behcet's disease. Forty observations in a cohort of 300 patients. J Mal Vasc. 2002;27(2):77-81.

19. Joseph FG, Scolding NJ. Neuro-Behçet's disease in Caucasians: a study of 22 patients. Eur J Neurol. 2007;14(2):174-180.

20. Kalra S, Silman A, Akman-Demir G, et al. Diagnosis and management of neuro-Behçet's disease: international consensus recommendations. J Neurol. 2014;261(9):1662-1676.

21. Gilman S, Wenning GF, Low PA, et al. Second consensus statement on the diagnosis of multiple system atrophy. Neurology. 2008;71(9): 670-676.

22. Marshall SE. Behçet's disease. Best Pract Res Clin Rheumatol. 2004;18(3):291-311.

23. Tugal-Tutkun I, Onal S, Altan-Yaycioglu R, Huseyin Altunbas H, Urgancioglu M. Uveitis in Behçet disease: an analysis of 880 patients. Am J Ophthalmol. 2004;138(3):373-380.

24. Kaçmaz RO, Kempen JH, Newcomb C, et al. Ocular inflammation in Behçet disease: incidence of ocular complications and of loss of visual acuity. Am J Ophthalmol. 2008;146(6):828-836.

25. George RK, Chan CC, Whitcup SM, Nussenblatt RB. Ocular immunopathology of Behçet's disease. Surv Ophthalmol. 1997;42(2):157-162.

26. Winter FC, Yukins RE. The ocular pathology of Behcet's disease. Am J Ophthalmol. 1966;62(2):257-262.

27. Evereklioglu C. Current concepts in the etiology and treatment of Behçet disease. Surv Ophthalmol. 2005;50(4):297-350.

28. Elgin U, Berker N, Batman A. Incidence of secondary glaucoma in Behcet's disease. J Glaucoma. 2004;13(6):441-444.

29. Kaklamani VG, Kaklamanis PG. Treatment of Behcet's disease - an update. Semin Arthritis Rheum. 2001;30(5):299-312.

30. Houman MH, Hamzaoui K. Promising new therapies for Behcet's disease. Eur J Intern Med. 2006;17(3):163-169.

31. Kidd D, Steuer A, Denman AM, Rudge P. Neurological complications in Behçet's syndrome. Brain. 1999;122(11):2183-2194.

32. Houman MH, Hamzaoui-B'Chir S, Ben Ghorbel I, et al. Neurologic manifestations of Behcet's disease: analysis of a series of 27 patients. Rev Med Intern. 2002;23(7):592-606. French.

33. Al-Araji A, Sharquie K, Al-Rawi Z. Prevalence and patterns of neurological involvement in Behçet's disease: a prospective study from Iraq. J Neurol Neurosurg Psychiatry. 2003;74(5):608-613.

34. Coban O, Bahar S, Akman-Demir G, et al. Masked assessment of MRI findings: is it possible to differentiate neuro-Behçet's disease from other central nervous system. Neuroradiology. 1999;41(4):255-260.
International Medical Case Reports Journal

\section{Publish your work in this journal}

The International Medical Case Reports Journal is an international, peer-reviewed open-access journal publishing original case reports from all medical specialties. Previously unpublished medical posters are also accepted relating to any area of clinical or preclinical science. Submissions should not normally exceed 2,000 words or

\section{Dovepress}

4 published pages including figures, diagrams and references. The manuscript management system is completely online and includes a very quick and fair peer-review system, which is all easy to use. Visit $\mathrm{http}: / /$ www.dovepress.com/testimonials.php to read real quotes from published authors. 